\title{
Preliminary Communication
}

\author{
Massimiliano Lamberto*
}

\section{Microwave-assisted synthesis of $\gamma$-thiolactams from ethyl isocyanoacetate}

https://doi.org/10.1515/hc-2018-0005

Received January 14, 2018; accepted March 6, 2018; previously published online April 21, 2018

Abstract: $\gamma$-Thiolactams were synthesized in good yields from ethyl isocyanoacetate through a sequential alkylation/isothiocyanation/radical cyclization under microwave irradiation.

Keywords: isocyanoacetate; isothiocyanate; microwave irradiation; radical cyclization; thiolactam.

Thioamides and thiolactams are important intermediates in organic synthesis [1-3], in particular, for gaining access to heterocycles of natural products [4-7]. Several procedures have been reported for the synthesis of thiolactams by conversion of the corresponding lactams; however, direct preparation of these compounds is not common $[8$, 9]. In this communication, we present an improved strategy for the synthesis of $\gamma$-thiolactams from simple isocyanoacetates [10] using microwaves (MWs)-assisted flash heating technology. In the early 1990s, Bachi reported $n-\mathrm{Bu}_{3} \mathrm{SnH}$ mediated radical cyclization of alkenyl isothiocyanates to give thiolactams (Scheme 1) [8, 11]. The mechanism of this reaction involves addition of organotin radical to isothiocyanate $\mathbf{1}$ to generate a tin thioimidoyl radical 2 which undergoes 5-exo cyclization onto an alkenyl side chain to give a tin thioimidate $\mathbf{3}$, which is spontaneously hydrolyzed during chromatography to give thiolactam 4.

Our interest in isocyanide radical chemistry and novel applications of MW technology to organic synthesis [12-14] led us to investigate the feasibility of using MWassisted synthesis to Bachi's approach to thiolactams. Our synthetic strategy first entailed the synthesis of alkenyl isothiocyanates that could be accessed by sequential reaction of an isocyanoacetic acid ester with alkenyl halides, followed by a reaction with tert-butyl mercaptan. A subsequent reaction with organotin reagents would give functionalized thiolactams (Scheme 2).

*Corresponding author: Massimiliano Lamberto, Department of Chemistry and Physics, Monmouth University, West Long Branch, NJ 07764, USA, e-mail: mlambert@monmouth.edu
We first synthesized alkenyl isocyanide 5 from commercially available ethyl isocyanoacetate and cinnamyl bromide using 2-tert-butylimino-2-diethylamino1,3-dimethylperhydro-1,3,2-diazaphosphorine (BEMP) as a base. The use of MWs afforded the desired compound in $10 \mathrm{~min}$ in $70 \%$ yield. Compound $\mathbf{5}$ was then allowed to react with tert-butyl thiol under MW irradiation to give the corresponding isothiocyanate $\mathbf{6}$ in 90\% yield (Scheme 2). With isothiocyanate 6 in hand, it was then possible to perform the radical cyclization in the presence of tributyltin hydride which gave $\gamma$-thiolactams 7 and $\mathbf{8}$ in $78 \%$ isolated yield [1.6:1 mixture of diastereoisomers by proton nuclear magnetic resonance ( $\left.\left.{ }^{1} \mathrm{H} \mathrm{NMR}\right)\right]$. This reaction was also accomplished in $6 \mathrm{~min}$ at $140^{\circ} \mathrm{C}$ in the MW synthesizer. The high yield conversion of the isocyanide to isothiocyanate and the radical cyclization prompted us to perform a two-step one-pot conversion of alkenyl isocyanide $\mathbf{5}$ to the corresponding thiolactam without isolating the isothiocyanate. The reactions were performed in the same MW vessel and gave the desired thiolactams 7 and 8 in 76\% overall yield (2:1 mixture of diastereoisomers by ${ }^{1} \mathrm{H}$ NMR). To further test this methodology, we then synthesized and reacted isocyanides $\mathbf{9}$ and $\mathbf{1 2}$ under the above conditions $[11,13]$. Conversion of allyl isocyanide 9 directly to thiolactams $\mathbf{1 0}$ and $\mathbf{1 1}$ worked well and the desired products were obtained in high isolated yields (1:1 mixture of diastereoisomers by NMR). The reaction was completed in $12 \mathrm{~min}$ without isolation of the isothiocyanate (Scheme 3).

When bis-allylated isocyanide $\mathbf{1 2}$ was treated with tertbutyl thiol and $n-\mathrm{Bu}_{3} \mathrm{SnH}$ under standard MW conditions,

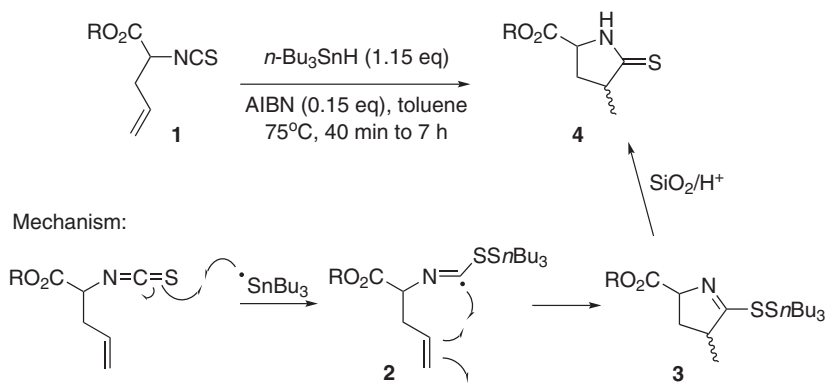

Scheme 1 Bachi's synthesis of thiolactams from isothiocyanates. 
<smiles>CCOC(=O)C1CC(Cc2ccccc2)C(=S)N1</smiles>

Scheme 2 Reagents and conditions: (i) cinnamyl bromide, BEMP, acetonitrile, MW 10 min at $110^{\circ} \mathrm{C}$; (ii) $t$-BuSH (1.5 eq), azobisisobutyronitrile (AIBN) $(0.2 \mathrm{eq})$, toluene, MW $6 \mathrm{~min}$ at $140^{\circ} \mathrm{C}$; (iii) $n-\mathrm{Bu}_{3} \mathrm{SnH}(1.15 \mathrm{eq})$, AIBN (0.2 eq), toluene, MW $6 \mathrm{~min}$ at $140^{\circ} \mathrm{C}$.<smiles>C=CCC(C=C)C(=O)OCC</smiles>

9

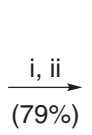<smiles>CCOC(=O)C1CC(C)C(=S)N1</smiles>

10,11
Scheme 3 Reagents and conditions: (i) $t$-BuSH (1.5 eq), AIBN (0.2 eq), toluene, MW 6 min at $140^{\circ} \mathrm{C}$; (ii) $n$-Bu $\mathrm{SnH}(1.15 \mathrm{eq})$, AIBN (0.2 eq), toluene, $\mathrm{MW} 6 \mathrm{~min}$ at $140^{\circ} \mathrm{C}$.<smiles>C=CCC(CC=C)(CC=C)C(=O)OCC</smiles>

12

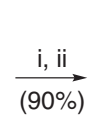<smiles>C/C=C\C[C@]1(C(=O)OCC)C[C@H](C)[C@@H](CC)N1</smiles>

13,14
Scheme 4 Reagents and conditions: (i) $t$-BuSH (1.5 eq), AIBN $(0.2$ eq), toluene, MW 6 min at $140^{\circ} \mathrm{C}$; (ii) $n-\mathrm{Bu}_{3} \mathrm{SnH}$ (1.15 eq), AIBN (0.2 eq), toluene, MW $6 \mathrm{~min}$ at $140^{\circ} \mathrm{C}$.

the corresponding thiolactams $\mathbf{1 3}$ and $\mathbf{1 4}$ were obtained in 90\% yield as a 1:1 diastereomeric mixture (Scheme 4).

In conclusion, MW flash heating methodology was successfully employed to quickly and efficiently synthesize $\gamma$-thiolactams in a two-step one-pot procedure in good yields from alkenyl isocyanides that are accessible from commercially available ethyl isocyanoacetate. This methodology additionally provides quick access to isothiocyanates from isocyanides by MW-assisted radical reaction with tert-butyl thiol.

\section{Experimental}

Flash column chromatography was performed using Sorbsil C60 (40-60 mesh silica gel). Dichloromethane and toluene were distilled from calcium hydride. ${ }^{1} \mathrm{H}$ NMR $(400 \mathrm{MHz})$ and carbon-13 nuclear magnetic resonance $\left({ }^{13} \mathrm{C} \mathrm{NMR}\right)(100 \mathrm{MHz})$ spectra were obtained in $\mathrm{CDCl}_{3}$ on a Bruker AVANCE III spectrometer. Infrared (IR) spectra were recorded on a Fourier-transform infrared spectroscopy (FTIR) Perkin-Elmer 2000 spectrometer coupled with an AutoIMAGE FTIR microscope. Mass spectrometry data were obtained on a ThermoQuest gas chromatograph-mass spectrometer configured for open access, on a Waters ZMD mass-spectrometer, single quadrupole, liquid chromatography-mass spectrometry (LCMS) and a VG analytical 70-250-SE double-focusing mass spectrometer. MW reactions were performed on a CEM MW synthesizer.

\section{Ethyl 2-isocyano-5-phenyl-pent-4-enoate (5)}

To a thick Pyrex tube were added ethyl isocyanoacetate $(250 \mu \mathrm{L}$, $2.17 \mathrm{mmol}$ ), cinnamyl bromide (450 mg, $2.17 \mathrm{mmol}$ ), BEMP (600 $\mu \mathrm{L}, 2.17 \mathrm{mmol}$ ) and $3.0 \mathrm{~mL}$ of dry acetonitrile. The tube was then flushed with nitrogen and capped. Heating was applied by means of microwave irradiation at $110^{\circ} \mathrm{C}$ for $10 \mathrm{~min}$. The tube was then cooled and the solvent was removed under reduced pressure. Purification by flash chromatography eluting with hexane/ethyl acetate (7:1) afforded compound 5: yield $348 \mathrm{mg}(70 \%) ;{ }^{1} \mathrm{H}$ NMR $\left(\mathrm{CDCl}_{3}\right): \delta 1.3(3 \mathrm{H}$, t, $\left.J=7.0 \mathrm{~Hz}, \mathrm{CH}_{3} \mathrm{CH}_{2} \mathrm{O}\right), 2.65-2.95\left(2 \mathrm{H}, \mathrm{m}, \mathrm{CHCH}_{2}\right), 4.3(3 \mathrm{H}, \mathrm{q}, J=7.0 \mathrm{~Hz}$, $\left.\mathrm{CH}_{2} \mathrm{O}\right), 4.4(1 \mathrm{H}, \mathrm{t}, J=7.0 \mathrm{~Hz}, \mathrm{CHN}) 6.1-6.25(1 \mathrm{H}, \mathrm{m}, \mathrm{CH}=\mathrm{CHPh}), 6.6(1 \mathrm{H}$, d, $J=16.0 \mathrm{~Hz}, \mathrm{PhCH}=\mathrm{CH}), 7.2-7.4$ (5H, m, PhH); ${ }^{13} \mathrm{C}$ NMR $\left(\mathrm{CDCl}_{3}\right): 14.4$, 36.8, 53.8, 57.0, 63.1, 121.9, 126.8, 128.3, 129.0, 135.7, 136.8, 161.0, 166.4; IR (neat): $v_{\max } 2139,1742,1250,1209,1060 \mathrm{~cm}^{-1}$; GC/MS (CI): $\mathrm{m} / z 230$ $\left(85 \%,[\mathrm{M}+\mathrm{H}]^{+}\right) ; 247\left(47 \%,\left[\mathrm{M}+\mathrm{NH}_{4}\right]^{+}\right)$. HRMS (EI). Calcd for $\mathrm{C}_{14} \mathrm{H}_{15} \mathrm{NO}_{2}$, $\mathrm{M}^{+}: m / z$ 229.11042. Found: $m / z 229.11028$.

\section{Ethyl 2-isothiocyanato-5-phenylpent-4-enoate (6)}

A thick Pyrex tube was charged with dry toluene $(2.0 \mathrm{~mL})$, compound 5 (176 mg, $0.769 \mathrm{mmol})$, tert-butyl thiol (112 $\mu \mathrm{L}, 1.0 \mathrm{mmol})$ and AIBN (23 mg, $0.146 \mathrm{mmol}$ ). The tube was flushed with argon and capped. Heating was then applied by means of microwave irradiation at $140^{\circ} \mathrm{C}$ for $6 \mathrm{~min}$. After cooling, the mixture was concentrated and the residue was subjected to column chromatography eluting with dichloromethane. The isothiocyanate $\mathbf{6}$ was obtained as a brownish liquid; yield $180 \mathrm{mg}(90 \%) ;{ }^{1} \mathrm{H} \mathrm{NMR}\left(\mathrm{CDCl}_{3}\right): \delta 1.2\left(3 \mathrm{H}, \mathrm{t}, J=7.0 \mathrm{~Hz}, \mathrm{CH}_{3} \mathrm{CH}_{2} \mathrm{O}\right)$, 2.7-2.9 (2H, m, $\left.\mathrm{CHCH}_{2} \mathrm{CH}=\mathrm{CH}\right), 4.18\left(3 \mathrm{H}, \mathrm{q}, J=7.0 \mathrm{~Hz}, \mathrm{CH}_{3} \mathrm{CH}_{2} \mathrm{O}\right)$, $4.25(1 \mathrm{H}, \mathrm{t}, J=7.0 \mathrm{~Hz}, \mathrm{CHN}) 6.0-6.1(1 \mathrm{H}, \mathrm{m}, \mathrm{CH}=\mathrm{CHPh}), 6.48(1 \mathrm{H}, \mathrm{d}$, $J=14.0 \mathrm{~Hz}, \mathrm{PhCH}), 7.1-7.3$ (5H, m, $\mathrm{PhH}) ;{ }^{13} \mathrm{C} \mathrm{NMR}\left(\mathrm{CDCl}_{3}\right): 14.3,37.4$, 59.6, 62.7, 122.4, 126.5, 127.9, 128.7, 135.4, 136.7, 168.0; IR (neat): $v_{\max }$ 2049, 1743, 1199, $1023 \mathrm{~cm}^{-1}$; GC/MS (CI): $\left.m / z 262(66 \% \text {, [M+H] }]^{+}\right) ; 279$ $\left(18 \%,\left[\mathrm{M}+\mathrm{NH}_{4}\right]^{+}\right)$; HRMS (EI). Calcd for $\mathrm{C}_{14} \mathrm{H}_{15} \mathrm{NO}_{2} \mathrm{~S}, \mathrm{M}^{+}: m / z 261.08235$. Found $m / z 261.08235$.

\section{Mixture of ethyl cis-4-benzyl-5-thioxopyrrolidine-2- carboxylate (7) and ethyl trans-4-benzyl-5-thioxo- pyrrolidine-2-carboxylate (8)}

A mixture of anhydrous toluene $(2 \mathrm{~mL})$, compound 6 (128 mg, $0.559 \mathrm{mmol})$, tri- $n$-butyltin hydride $(46.2 \mu \mathrm{L}, 0.166 \mathrm{mmol})$ and AIBN (3.6 mg, $0.022 \mathrm{mmol}$ ) in a sealed Pyrex tube under argon atmosphere was heated by means of MW irradiation at $140^{\circ} \mathrm{C}$ for $6 \mathrm{~min}$. After cooling, the solvent was removed under reduced pressure and the residue was subjected to silica gel chromatography eluting with 
hexane/ethyl acetate (7:1) to afford compounds 7 and $\mathbf{8}$ as an inseparable diastereomeric mixture in the ratio of 1.6:1; yield $22.6 \mathrm{mg}(78 \%)$; ${ }^{1} \mathrm{H}$ NMR $\left(\mathrm{CDCl}_{3}\right)$ for 7: $\delta 1.19\left(3 \mathrm{H}, \mathrm{t}, J=7.0 \mathrm{~Hz}, \mathrm{CH}_{3} \mathrm{CH}_{2} \mathrm{O}\right), 1.90(1 \mathrm{H}, \mathrm{dt}$, $J=13.0,9.0 \mathrm{~Hz}, \mathrm{NCHCHH}), 2.43(1 \mathrm{H}, \mathrm{dt}, 13.0,8.0 \mathrm{~Hz}, \mathrm{NCHCHH}), 2.52$ $(1 \mathrm{H}, \mathrm{dd}, J=14.0,10.5 \mathrm{~Hz}, \mathrm{PhCH} H), 3.0\left(1 \mathrm{H}, \mathrm{m}, \mathrm{PhCH}_{2} \mathrm{CH}\right), 3.56(1 \mathrm{H}$, dd, $J=14.0,4.0 \mathrm{~Hz}, \mathrm{PhCHH}), 4.04$ (1H, dd, $J=9.0,5.0 \mathrm{~Hz}, \mathrm{CHN}), 4.14$ $\left(2 \mathrm{H}, \mathrm{q}, J=7.0 \mathrm{~Hz}, \mathrm{CH}_{2} \mathrm{O}\right), 7.82(1 \mathrm{H}$, br s, $\mathrm{NH})$; ${ }^{1} \mathrm{H} \mathrm{NMR}\left(\mathrm{CDCl}_{3}\right)$ for 8 : $\delta 1.21\left(3 \mathrm{H}, \mathrm{t}, J=7.0 \mathrm{~Hz}, \mathrm{CH}_{3} \mathrm{CH}_{2} \mathrm{O}\right), 2.15-2.30(2 \mathrm{H}, \mathrm{m}, \mathrm{NCHCHH}), 2.69$ (1H, dd, $J=13.5,9.5 \mathrm{~Hz}, \mathrm{PhCH}), 3.07-3.15\left(1 \mathrm{H}, \mathrm{m}, \mathrm{PhCH}_{2} \mathrm{CH}\right), 3.36$ $(1 \mathrm{H}, \mathrm{dd}, J=13.5,4.0 \mathrm{~Hz}, \mathrm{PhCHH}), 4.11\left(2 \mathrm{H}, \mathrm{q}, J=7.0 \mathrm{~Hz}, \mathrm{CH}_{2} \mathrm{O}\right), 4.30$ (1H, t, $J=8.0 \mathrm{~Hz}, \mathrm{CHN}), 7.70$ (1H, br s, NH); GC/MS (EI): $m / z 263\left(\mathrm{M}^{+}\right)$, $91\left(\mathrm{PhCH}_{2}^{+}\right)$. HRMS (EI). Calcd for $\mathrm{C}_{14} \mathrm{H}_{17} \mathrm{NO}_{2} \mathrm{~S},[\mathrm{M}]^{+}: \mathrm{m} / z 263.09800$. Found: $m / z 263.09789$.

\section{Ethyl-2-isocyanopent-4-enoate (9)}

A mixture of ethyl isocyanoacetate ( $3.0 \mathrm{~mL}, 25.8 \mathrm{mmol}$ ), allyl bromide (2.21 mL, $25.8 \mathrm{mmol})$, tetra- $N$-butylammonium bromide $(832.6 \mathrm{mg}$, $2.59 \mathrm{mmol})$, finely ground potassium carbonate $(10.7 \mathrm{~g}, 77.5 \mathrm{mmol})$ and acetonitrile $(50 \mathrm{~mL})$ was heated under reflux with stirring for $20 \mathrm{~h}$. The mixture was then cooled, filtered and the solution was concentrated in vacuo. The resultant brownish oil was subjected to silica gel chromatography eluting with hexane/ethyl acetate (7:1). Compound 9 was obtained as a yellow oil; yield $2.38 \mathrm{~g}(62 \%)$; ${ }^{1} \mathrm{H}$ NMR $\left(\mathrm{CDCl}_{3}\right): 1.3\left(3 \mathrm{H}, \mathrm{t}, J=7.0 \mathrm{~Hz}, \mathrm{CH}_{3} \mathrm{CH}_{2} \mathrm{O}\right), 2.6-2.7\left(2 \mathrm{H}, \mathrm{m}, \mathrm{CHCH}_{2}\right), 4.23$ $\left(2 \mathrm{H}, \mathrm{q}, J=7.0 \mathrm{~Hz}, \mathrm{CH}_{2} \mathrm{O}\right), 4.3(1 \mathrm{H}, \mathrm{t}, J=5.0 \mathrm{~Hz}, \mathrm{NCH}), 5.2-5.3(2 \mathrm{H}, \mathrm{m}$, $\left.\mathrm{CH}=\mathrm{CH}_{2}\right), 5.7-5.9\left(1 \mathrm{H}, \mathrm{m}, \mathrm{CH}=\mathrm{CH}_{2}\right) ;{ }^{13} \mathrm{C} \mathrm{NMR}\left(\mathrm{CDCl}_{3}\right): 14.1,37.1,56.4$, $62.8,120.6,130.5,160.3,166.2$; IR (neat): $v_{\max } 2145,1753 \mathrm{~cm}^{-1}$.

\section{Mixture of ethyl cis-4-methyl-5-thioxo-pyrrolidine- 2-carboxylate (10) and trans-4-methyl-5-thioxo- pyrrolidine-2-carboxylate (11)}

These compounds were obtained by using the procedure described earlier for the mixture of $\mathbf{7}$ and $\mathbf{8}$. Purification by silica gel chromatography eluting with hexane/ethyl acetate (8:1) afforded compounds $\mathbf{1 0}$ and $\mathbf{1 1}$ as an inseparable 1:1 diastereomeric mixture; yield $115 \mathrm{mg}$ (79\%); ${ }^{1} \mathrm{H} \mathrm{NMR}\left(\mathrm{CDCl}_{3}\right)$ for 10: $\delta 1.22\left(3 \mathrm{H}, \mathrm{d}, J=7.0 \mathrm{~Hz}, \mathrm{CH}_{3} \mathrm{CH}\right), 1.30$ (3H, t, $\left.J=7.0 \mathrm{~Hz}, \mathrm{CH}_{3} \mathrm{CH}_{2} \mathrm{O}\right), 1.89(1 \mathrm{H}, \mathrm{dt}, J=12.0,9.0 \mathrm{~Hz}, \mathrm{NCHCHH})$, $2.76(1 \mathrm{H}, \mathrm{dt}, 12.0,8.0 \mathrm{~Hz}, \mathrm{NCHCHH}), 2.80-2.97\left(1 \mathrm{H}, \mathrm{m}, \mathrm{CH}_{3} \mathrm{CH}\right), 4.23$ $\left(2 \mathrm{H}, \mathrm{q}, J=7.0 \mathrm{~Hz}, \mathrm{CH}_{2} \mathrm{O}\right), 4.4(1 \mathrm{H}, \mathrm{m}, \mathrm{CHN}), 8.0(1 \mathrm{H}, \mathrm{br} \mathrm{s}, \mathrm{NH}) ;{ }^{1} \mathrm{H}$ NMR $\left(\mathrm{CDCl}_{3}\right)$ for 11: $\delta 1.22\left(3 \mathrm{H}, \mathrm{d}, J=7.0 \mathrm{~Hz}, \mathrm{CH}_{3} \mathrm{CH}\right), 1.30(3 \mathrm{H}, \mathrm{t}, J=7.0 \mathrm{~Hz}$, $\left.\mathrm{CH}_{3} \mathrm{CH}_{2} \mathrm{O}\right), 2.15\left(1 \mathrm{H}, \mathrm{dt}, J=13.0,7.5 \mathrm{~Hz}, \mathrm{CH}_{3} \mathrm{CHCHH}\right), 2.54-2.62(1 \mathrm{H}, \mathrm{m}$, $\left.\mathrm{fCH}_{3} \mathrm{CHCHH}\right), 2.80-2.97\left(1 \mathrm{H}, \mathrm{m}, \mathrm{CH}_{3} \mathrm{CH}\right), 4.23\left(2 \mathrm{H}, \mathrm{q}, J=7.0 \mathrm{~Hz}, \mathrm{CH}_{2} \mathrm{O}\right)$, $4.4(1 \mathrm{H}, \mathrm{m}, \mathrm{CHN}), 8.0(1 \mathrm{H}, \mathrm{br} \mathrm{s}, \mathrm{NH})$; ${ }^{13} \mathrm{C} \mathrm{NMR}\left(\mathrm{CDCl}_{3}\right)$ for the mixture: $\delta 14.5,19.2,19.3,35.2,35.5,46.7,47.4,60.2,60.3,62.4,170.3,170.5$, 211.2; GC/MS (EI) for the mixture: $m / z 188,\left[\mathrm{M}+\mathrm{H}^{+}\right.$; HRMS (EI) for the mixture. Calcd for $\mathrm{C}_{8} \mathrm{H}_{13} \mathrm{NO}_{2} \mathrm{~S}$, [M] $]^{+}: m / z$ 187.06670. Found: $m / z$ 187.06715.

\section{Mixture of ethyl 2-allyl-cis-4-methyl-5-thioxo-pyrroli- dine-2-carboxylate (13) and ethyl 2-allyl-trans-4-methyl- 5-thioxo-pyrrolidine-2-carboxylate (14)}

A mixture of compound 12 (108 mg, $0.559 \mathrm{mmol})$, tert-butyl thiol $(81.5 \mu \mathrm{L}, 0.727 \mathrm{mmol})$ and AIBN $(17.5 \mathrm{mg}, 0.111 \mathrm{mmol})$ in $2.0 \mathrm{~mL}$ of dry toluene in a sealed Pyrex tube under an argon atmosphere was microwave-irradiated at $140^{\circ} \mathrm{C}$ for $6 \mathrm{~min}$. After cooling, the mixture in the tube was treated with tri- $n$-butyltin hydride $(170 \mu \mathrm{L}, 0.614 \mathrm{mmol})$ and AIBN (23 mg), flushed with argon, capped and heated again at $140^{\circ} \mathrm{C}$ for $6 \mathrm{~min}$ as described earlier. After removal of the solvent under reduced pressure, the residue was subjected to silica gel chromatography eluting with hexane/ethyl acetate (7:1). Compounds 13 and $\mathbf{1 4}$ were obtained as an inseparable diastereomeric mixture in the ratio of 1:1; yield $113 \mathrm{mg}(90 \%)$; GC/MS (EI): $\mathrm{m} / z 227$ (86\%, [M]+, 154 (96\%). HRMS (ESI). Calcd for $\mathrm{C}_{11} \mathrm{H}_{18} \mathrm{NO}_{2} \mathrm{~S},[\mathrm{M}+\mathrm{H}]^{+}: m / z 228.1053$. Found: $m / z$ 228.1055.

Acknowledgments: The author would like to thank Professor Jeremy D. Kilburn for providing useful insights and comments that greatly assisted the research and Monmouth University for funding.

\section{References}

[1] Ilankumaran, P.; Ramesha, A. R.; Chandrasekaran, S. A facile conversion of amides and lactams to thioamides and thiolactams using tetrathiomolybdate. Tetrahedron Lett. 1995, 35, 8311-8314.

[2] Smith, D. C.; Lee, S. W.; Fuchs, P. L. Conversion of amides and lactams to thioamides and thiolactams using hexamethyldisilathiane. J. Org. Chem. 1994, 59, 348-354.

[3] Okada, T.; Minehira, D.; Takada, M.; Urata, H.; Kato, A.; Adachi, I.; Kurashima, Y.; Kaji, S.; Ogura, T.; Chiba, S.; et al. Synthesis of new tricyclic thiolactams as potent antitumor agent for pancreatic cancer. Bioorg. Med. Chem. Lett. 2016, 26, 2577-2579.

[4] Heathcock, C. H.; Davidsen, S. K.; Mills, S. G.; Sanner, M. A. J. Daphniphyllum alkaloids. 10. Classical total synthesis of methyl homodaphniphyllate. J. Org. Chem. 1992, 57, 2531-2544.

[5] Magnus, P.; Mendoza,J. S.; Stamford, A.; Ladlow, M.; Willis, P. Nonoxidative coupling methodology for the synthesis of the antitumor bisindole alkaloid vinblastine and a lower-half analog: solvent effect on the stereochemistry of the crucial C-15/C-18' bond. J. Am. Chem. Soc. 1992, 114, 10232-10245.

[6] Abell, A. D.; Prince, M. J.; McNulty, A. M.; Neubauerb, B. L. Simple bi- and tricyclic inhibitors of human steroid $5 \alpha$-reductase. Bioorg. Med. Chem. Lett. 2000, 10, 1909-1911.

[7] Bachi, M. D.; Bar-Ner, N.; Melman, A. Stereoselective synthesis of $\alpha$-kainic acid using free radical key reactions. J. Org. Chem. 1996, 61, 7116-7124.

[8] Bachi, M. D.; Denenmark, D. Intramolecular addition of carboncentered tinthioimidoyl radicals to carbon-carbon double bonds. Synthesis of $\gamma$-and $\delta$-thiolactams. J. Org. Chem. 1990, 55, 3442-3444.

[9] Cho, M. S.; Lee, I. S.; Kang, S. H.; Kim, Y. H. Intramolecular, reductive cyclization of b-ketoisothiocyanates promoted by using samarium diiodide. Chem. Eur. J. 2005, 11, 1452-1458.

[10] Gulevich, A. V.; Zhdanko, A. G.; Orru, R. V. A.; Nenajdenko, V. G. Isocyanoacetate derivatives: synthesis, reactivity, and application. Chem. Rev. 2010, 110, 5235-5331.

[11] Bachi, M. D.; Melman, A. Stereocontrolled 5-exo-trig cyclization of imidoyl radicals in the synthesis of substituted (alkylthio) pyrrolines, pyroglutamates, and thiopyroglutamates. J. Org. Chem. 1995, 60, 6242-6244. 
[12] Lamberto, M.; Corbett, D. F.; Kilburn, J. D. Microwave assisted free radical cyclization of alkenyl and alkynyl isocyanides with thiols. Tetrahedron Lett. 2003, 44, 1347.

[13] Lamberto, M.; Kilburn, J. D. Synthesis of indolizidines from dialkylated isocyanides: a novel radical cyclization/ $\mathrm{N}$-alkyla- tion/ring closing metathesis approach. Tetrahedron Lett. 2008, 49, 6364-6367.

[14] Lamberto, M. Synthesis of tricyclic indolizidines from ethyl isocyanoacetate. Heterocycl. Commun. 2015, 21, 153-157. 